\title{
Progressive Supranuclear Palsy: A Crucial Co-occurrence in Normal Pressure Hydrocephalus or an Etiology of Hydrocephalic Presentation?
}

\author{
Halil Onder ${ }^{1}$ \\ ${ }^{1}$ Department of Neurology, Yozgat City Hospital, Yozgat, Turkey
}

\author{
Address for correspondence Halil Onder, MD, Department of \\ Neurology, Yozgat City Hospital, Yozgat 66100, Turkey \\ (e-mail: halilnder@yahoo.com).
}

\begin{abstract}
Co-occurrences of neurodegenerative diseases in normal pressure hydrocephalus (NPH) is a remarkable topic of discussion, which has been mentioned in recent studies and it was also emphasized by the International Society for Hydrocephalus and Cerebrospinal Fluid Disorder. In this report, I illustrate two remarkable cases which were initially diagnosed with idiopathic normal pressure hydrocephalus (iNPH) and ventriculoperitoneal (VP)

Keywords

- normal pressure hydrocephalus

- progressive supranuclear palsy

- neurodegenerative disease shunt surgery was performed, which yielded significant improvement in the clinic of both patients in the short-term period. However, in the long-term follow-up, both of them deteriorated, and re-evaluation of the neurological examination and neuroimaging data yielded the full clinical picture of progressive supranuclear palsy (PSP). Via the illustration of these cases and related literature data, I rediscuss some related hypotheses about the co-occurrence of these two conditions of hydrocephalus and PSP. I also suggest some distinct discussions to be clarified in future studies.
\end{abstract}

\section{Introduction}

Idiopathic normal pressure hydrocephalus (iNPH) is a neurological condition clinically characterized by the triad of gait dysfunction, cognitive abnormalities, and urinary incontinence. In addition to these clinical manifestations, the diagnosis can be made with the aid of neuroimaging evidence of ventriculomegaly by excluding secondary hydrocephalic disorders. However, it is a diagnosis without definitive pathological findings, and there are many pitfalls associated with the diagnosis of iNPH. ${ }^{1}$ First, the full clinical triad is not present in considerable number of patients; also, the components of this trial are separately nonspecific for the diagnosis of iNPH as they can be present in high rates of the general elderly population. ${ }^{2-4}$ Second, the comorbidity of a possible neurodegenerative disorder in normal pressure hydrocephalus (NPH) has been reported multiple times previously. The existence of these comorbidities in NPH has been defined as "dual"

DOI https://doi.org/

$10.1055 / \mathrm{s}-0040-1708080$

ISSN 2277-954X. pathology. However, some authors explained the existence of the high-incidence of the neurodegenerative disorders in NPH by a hypothesis that NPH may predispose to neurodegenerative diseases. ${ }^{5-7}$ On the other hand, there are also other authors suggesting the consideration that some neurodegenerative phenotypes may present with ventriculomegaly, which was proposed as "neurodegenerative $\mathrm{NPH}$ " in a recent crucial report by Espay et al. ${ }^{1,4}$ Remarkably, in their study, Espay et al suggested that the response to shunt surgery might not be sufficient to base the argument for diagnosis of a disease and they have reported a big rate of patients developing a neurodegenerative disorder in the long-term follow-up, leading to poor long-term postshunt benefits. They remarked to the lack of a randomized, placebo-controlled, double-blind clinical trial to show that the improvement seen after shunting procedures for patients with iNPH was present in the long-term follow-ups. In the light of these discussions, herein, I illustrate two remarkable cases who were initially diagnosed

(C) 2020. Neurological Surgeons' Society of India.

This is an open access article published by Thieme under the terms of the Creative Commons Attribution-NonDerivative-NonCommercial-License, permitting copying and reproduction so long as the original work is given appropriate credit. Contents may not be used for commercial purposes, or adapted, remixed, transformed or built upon. (https://creativecommons.org/licenses/by-nc-nd/4.0/)

Thieme Medical and Scientific Publishers Pvt. Ltd., A-12, 2nd Floor, Sector 2, Noida-201301 UP, India 
with iNPH. However, long-term follow-up of the patients revealed the final diagnosis of progressive supranuclear palsy (PSP). Via the illustration of these cases, I draw attention to this neurodegenerative pathology of PSP to be kept in mind in NPH-like presentations. I also postulate some crucial discussions regarding the association of these two entities as well as diagnostic pitfalls and definition of iNPH.

\section{Case 1}

A 75-year-old male patient was attended to due to walking impairment and urinary incontinence that had started over the previous 1 year. Upon questioning of the history, it was also learned that newly onset memory impairment was recognized during this period. On neurological examination, the patient was fully orientated and cooperative. Cranial nerve examinations revealed mildly slow saccades. Motor, sensory, and cerebellar examinations were within normal ranges. Extrapyramidal examinations revealed slight rigidity in the upper extremities. However, his walking was characterized with small steps and he experienced significant difficulty, particularly during turning which was compatible with severe lower body Parkinsonism. Besides, severe postural instability was found, such that the patient could not mobilize without a support and he had several falls over the last few months. Cranial tomography revealed enlarged ventricles, dilatated Sylvian fissures out of proportion to sulcal enlargement, and crowding of the gyri at the vertex which was compatible with NPH ( - Fig. 1A). The standardized mini-mental test (SMMT) examinations score was evaluated as 22 points. Lumbar puncture opening pressure was $16 \mathrm{~cm} \mathrm{H}_{2} 0$, and 40 $\mathrm{mL}$ cerebrospinal fluid (CSF) was drained, which yielded a significant improvement in the patient's walking and mobility. Ergo, the patient and his relatives were informed about the disease and ventriculoperitoneal shunt (VP) shunt was recommended. After obtaining approval, the VP shunt was inserted, which yielded a moderate improvement in this walking and urinary incontinence at evaluation soon after the operation. The patient could mobilize without support and perform activities of daily living at home. However, he was reattended to 6 months after the operation due to worsening of the symptoms over the past 2 months. With a preliminary consideration of malfunction of the shunt, he was hospitalized for performing the CSF tap test. However, the CSF tap test did not provide an improvement in the patient's clinic. Besides, the neurosurgeon evaluation confirmed that the VP shunt was working properly. Hence, the patient was discharged with suggestions of the physical therapy and rehabilitation department including balance exercises. Nevertheless, the patient did not attendthe routine polyclinic visits after discharge. However, neurological examination when he visited our center 3 years later revealed remarkable results. His neurological condition was found to worsen devastatingly. The patient was orientated; however, he was only moderately cooperative due to severe cognition impairment. The SMMT score was 10 . He could count three digits forward and could not count backward, showing severe impairment in attention and executive functions. Besides, significant deterioration in visuospatial functions was determined (he scored 0 in a clock drawing test and he could not copy objects). Cranial nerve examinations revealed severe disturbances in saccadic eye movements in all directions. In addition, vertical eye movement of the upright position was nearly totally restricted. As far as it can be assessed (due to cooperation problems and severe rigidity), motor and sensory examinations were within the normal ranges. Extrapyramidal examinations revealed severe rigidity and tonus increase in all of the extremities. Tonus changes in the upper extremities were compatible with oppositional paratonia (gegenhalten) and were evaluated as dystonic and spastic in the lower extremities. Severe postural instability was present such that the patient could not stand upright on his own. Cranial magnetic resonance imaging (MRI) showed overall, severe cerebral atrophy developing in the interval period. However, midbrain atrophy was prominent and sagittal T1 images revealed concave midbrain at its cranial margin, which was compatible with hummingbird sign. In - Fig. 1B-C, Cranial MRIs, at 19-month intervals, demonstrate the progressive brain stem atrophy. Taken together, the
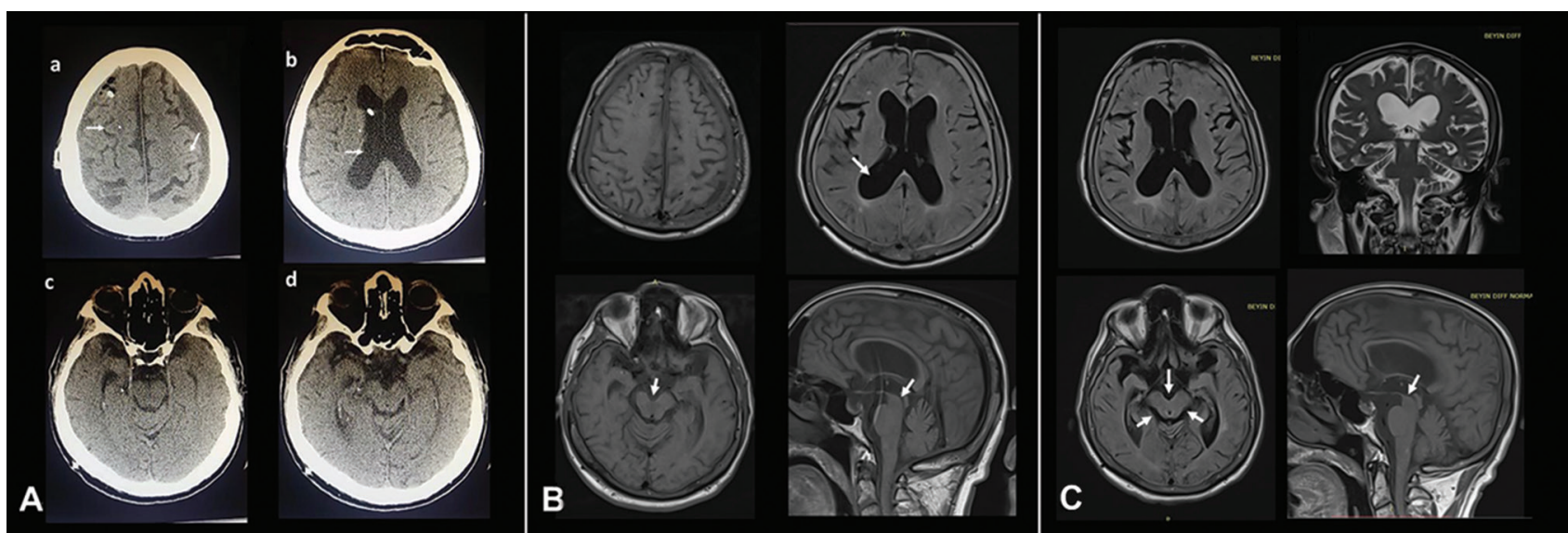

Fig. 1 (A), Cranial CT of patient 1 (recorded on 10.06.2016, 1 month after VP shunt), showing enlarged ventricles (b) and crowding of the gyri at the vertex (a), which were compatible with NPH. Brain stem atrophy was not substantive initially (c, d). B-C, cranial MRI images recorded on 16.08.2017 (B) and 17.03.2019 (C), showing progressive brain stem atrophy. CT, computed tomography; MRI, magnetic resonance imaging; $\mathrm{NPH}$, normal pressure hydrocephalus; VP, ventriculoperitoneal. 


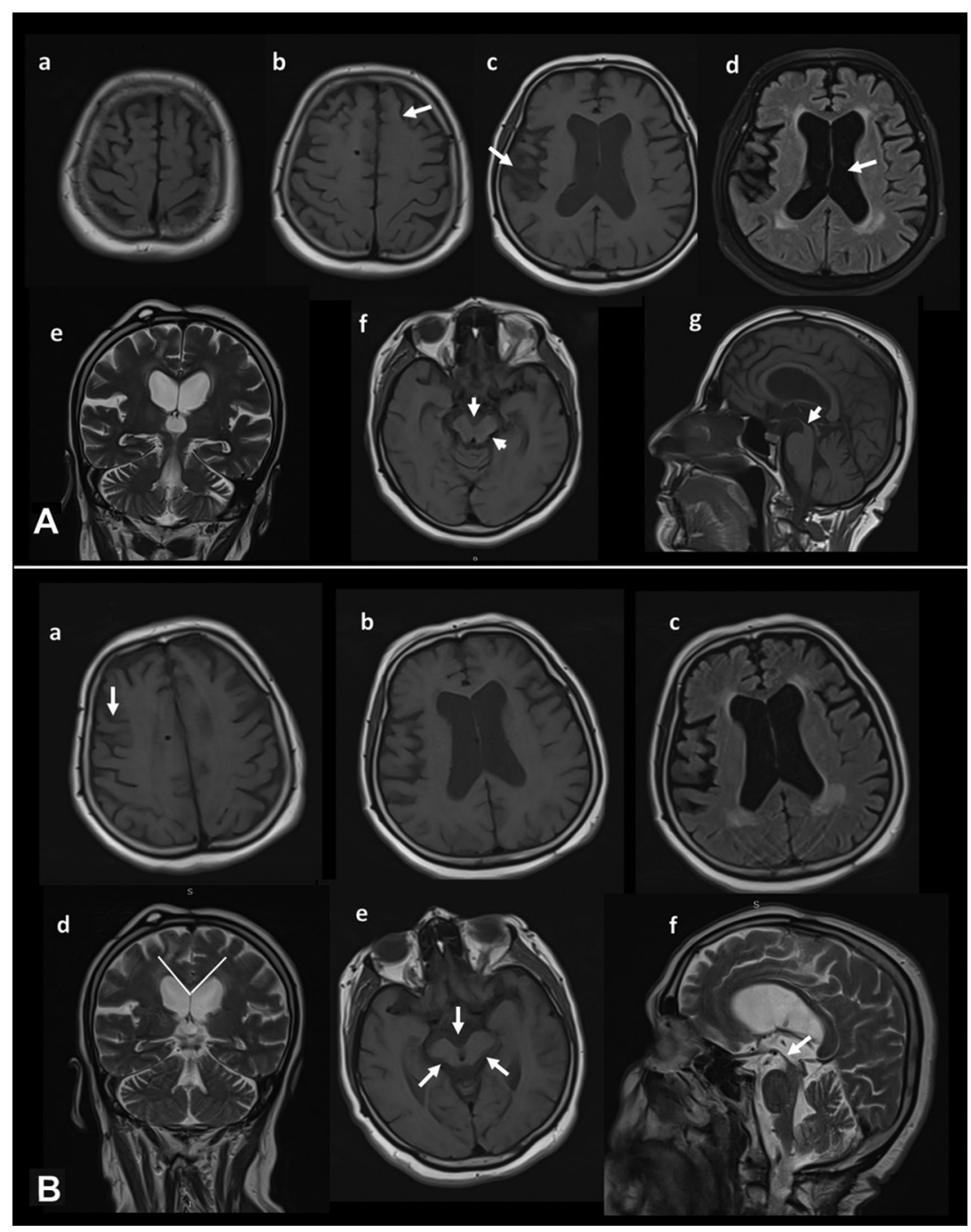

Fig. 2 (A) Enlarged ventricles (c, d), dilatated Sylvian fissures out of proportion to sulcal enlargement (c, d) and crowding of the gyri at the vertex (a, b). Moderate midbrain atrophy (e) and sagittal T1 images revealed concave midbrain at its cranial margin which was compatible with hummingbird sign (f). (B) Enlarged ventricles (b, c), dilatated Sylvian fissures out of proportion to sulcal enlargement (b, c) and progressive atrophy at the vertex in the interval period (a). Callosal narrowing (d), severe (progressive) midbrain atrophy (e), and sagittal T1 images revealed concave midbrain at its cranial margin which was compatible with hummingbird sign (f).

diagnosis of PSP was established, and the patient's relatives were informed about the disease and prognosis.

\section{Case 2}

A 64-year-old female patient was attended to due to progressive gait difficulty and recurring falls which had redeveloped in the past 2 years. Upon questioning, her medical history revealed that she was diagnosed with iNPH 4 years ago in the movement disorder clinic of a tertiary care hospital, based on the neuroimaging findings and positive CSF tap test response; besides, a clinic of progressive gait difficulty, and cognitive impairment developing in the last one-year period prior to the admission to their center. Ergo, a VP shunt was inserted which had provided a significant improvement in the patient's symptoms, such that she could mobilize without a support and perform some of her daily living activities without support (these were learned from the hospital discharge report). On neurological examination at admission to our clinic, she was found to be moderately orientated and cooperative and her SMMT score was 18. However, cranial nerve examinations revealed supranuclear gaze palsy. Extrapyramidal examinations revealed mild rigidity in the upper extremities and tonus increment in the lower extremities compatible with dystonia. On the other hand, the patient experienced severe postural instability and could not mobilize without support. Cranial MRI revealed severe midbrain atrophy and sagittal T1 images revealed concave midbrain at its cranial margin, which was compatible with hummingbird sign. In - Fig. 2A-B, Cranial MRIs, at 18-month intervals, are illustrated. PSP was diagnosed, nonetheless, based on the possible contributory role of progression of NPH pathophysiology (in the setting of the malfunction of VPshunt?); the CSF tap test was also performed which did not provide any improvement in the clinic. 


\section{Discussion}

In this report, I have illustrated the detailed clinic and neuroimaging findings of two patients presenting with NPH clinic and evidence of ventriculomegaly, who improved moderately in the acute period after VP shunt surgery. However, follow-up of the patients revealed the development of PSP clinically as well as the inclusion of neuroimaging signs. As far as we know, reports of detailed illustrations of these remarkable samples have not been reported to date. I would like to state that serial neuroimaging data, showing progressive atrophy of the midbrain in both of our patients, is strictly rare in literature. Hence, I think that the clinical presentations of these patients may provide substantial perspectives to be kept in mind in clinical practice.

NPH and PSP are distinct causes of Parkinsonism; however, they share some common clinical features including gait dysfunction, postural instability with retropulsion, and cognitive impairment. ${ }^{8}$ Besides, in terms of the underlying pathophysiology, dysfunction of the frontal lobe-basal ganglia and thalamo-mesencephalic loops have been suggested to be involved in both of the disease. ${ }^{8}$ However, although their clinical presentations may mimic each other in some circumstances, the classical knowledge regarding the perception of these diseases are strictly different. Such that, while NPH is evaluated as a condition of abnormality of CSF dynamics, PSP can be classified under the head of tau protein which is associated neurodegenerative diseases. On the other hand, the concurrences of NPH and neurogenerative diseases have been reported in recent reports were initially preferred to be defined as "dual" pathology. However, with the help of the recent reports including pathological examinations, many discussions and hypotheses have been suggested about this topic. ${ }^{1,9,10}$ For instance, Starr et al postulated that previously reported cases of "dual" pathology (e.g., NPH and PSP) actually represent the hydrocephalic presentation of selected neurodegenerative disorders. ${ }^{9}$ Moreover, in his crucial report, Saper remarked the controversies regarding the definition of iNPH..$^{10} \mathrm{He}$ mentioned that in many related studies on shunting for iNPH had required only two or even one of these symptoms to include the patient in the series. In addition, CSF pressure was not mentioned in many of them. In conclusion, he emphasized that the data regarding the response to surgery was the sole defining characteristic of this supposed disorder. However, in a recent report, Espay et al. ${ }^{1}$ summarized their long-term institutional experience, in which postshunt benefits in patients with initial diagnosis of idiopathic NPH persist in only $32 \%$ of patients at 36 months. They also reported that in over $25 \%$ of the patients, revised diagnoses of Alzheimer's disease, dementia with Lewy bodies, and PSP were made. Taken together, they suggested that the response to shunt surgery in the short-term might not be sufficient to base the argument for diagnosis of iNPH, which further complicated the knowledge regarding the NPH physiology. In accordance with these results, Magdalinou et al had reported remarkable results in conclusion of the postmortem pathological examinations of four patients who had received a diagnosis of iNPH during life. ${ }^{11}$ The pathological examinations had shown that one patient had Parkinson's disease (PD), while the other three had PSP. They drew attention to the importance of awareness that PSP or Parkinson's disease can mimic the clinical symptoms of iNPH to avoid invasive and futile CSF shunting procedures. Besides, the authors also remarked that the absence of urinary incontinence and the presence of a supranuclear gaze palsy in a suspected case of iNPH should lead to a reconsideration of the diagnosis and raise the possibility of the PAGF (pure akinesia with gait freezind) variant of PSP. I would like to state that in all of these patients reported, at least a transient response had been achieved by CSF diversion method (LP or VP shunt). Accordingly, VP shunt had also provided a short-term improvement in both patients from my experience. However, the clinical course of the patients worsened in the follow-up and the clinic of PSP had developed in the long-term course.

A major discussion may involve the potential role of VP shunt in the long-term in these patients with a final diagnosis of PSP. Remarkably, it is a known hypothesis that cognitive impairment in NPH might also be a result of a combination of CSF dynamic disturbance and its resulting biochemical abnormalities. ${ }^{12}$ In accordance with this view, a combination of $A B 1-42$ and total tau levels in ventricular CSF was found to predict favorable surgical outcomes with high-sensitivity and specificity in a large group of patients with $\mathrm{NPH} .{ }^{13}$ In another report, VP shunting in 35 patients with NPH had led to a reduction in CSF neurofilament protein. ${ }^{14}$ Ergo, it can also be hypothesized that VP shunt surgery might also influence positively the clinical course of PSP via a mechanism of reduction in CSF tau protein. It can also be considered that the VP shunt surgery might have a positive influence via eliminating the contributory effect of hydrocephalus in the clinical course of the patients, other than interacting with neurogenerative processes of PSP. Taken together, giving a suggestions of avoiding VP shunt surgery in this subgroup of hydrocephalic patients with PSP, as there is no randomized, controlled study showing inefficaciousness of VP shunting on long-term follow-up. However, surely, determination of this subgroup of patients of PSP presenting with hydrocephalus would be critical while predicting the prognosis of the patients as well as planning a possible shunt surgery in a more deliberative manner. More importantly, identification of these rare subgroups may provide substantial contributions to our understanding of the unknown aspects of NPH as well as possible distinct mechanisms underlying PSP. Future reports including large case series of these samples may give crucial perspectives in this regard. Finally, I also think that the possible utility of CSF diversion methods, even in the treatment of some neurodegenerative pathologies (without hydrocephalic presentations?), may constitute an interesting point of discussion to be clarified in these future studies.

\section{Funding}

None.

\section{Conflict of Interest}

None declared. 


\section{References}

1 Espay AJ, Da Prat GA, Dwivedi AK, et al. Deconstructing normal pressure hydrocephalus: ventriculomegaly as early sign of neurodegeneration. Ann Neurol 2017;82(4):503-513

2 Stothers L, Thom D, Calhoun E. Urologic diseases in America project: urinary incontinence in males-demographics and economic burden. J Urol 2005;173(4):1302-1308

3 Verghese J, Lipton RB, Hall CB, Kuslansky G, Katz MJ, Buschke $\mathrm{H}$. Abnormality of gait as a predictor of non-Alzheimer's dementia. N Engl J Med 2002;347(22):1761-1768

4 Espay AJ, Lang AE. Is there even such a thing as "idiopathic normal pressure hydrocephalus"? Ann Neurol 2017;82(6):1032

5 Malm J, Graff-Radford NR, Ishikawa M, et al. Influence of comorbidities in idiopathic normal pressure hydrocephalus research and clinical care. A report of the ISHCSF task force on comorbidities in INPH. Fluids Barriers CNS 2013;10(1):22

6 Koivisto AM, Alafuzoff I, Savolainen S, et al; Kuopio NPH Registry (, www.uef.finph. Poor cognitive outcome in shunt-responsive idiopathic normal pressure hydrocephalus. Neurosurgery 2013;72(1):1-8, discussion 8

7 Koivisto AM, Kurki MI, Alafuzoff I, et al. high risk of dementia in ventricular enlargement with normal pressure hydrocephalus related symptoms1. J Alzheimers Dis 2016;52(2):497-507

8 Selge C, Schoeberl F, Zwergal A, et al. Gait analysis in PSP and NPH: dual-task conditions make the difference. Neurology 2018;90(12):e1021-e1028
9 Starr BW, Hagen MC, Espay AJ. Hydrocephalic Parkinsonism: lessons from normal pressure hydrocephalus mimics. J Clin Mov Disord 2014;1:2

10 Saper CB. Is there even such a thing as "Idiopathic normal pressure hydrocephalus"? Ann Neurol 2017;82(4):514-515

11 Magdalinou NK, Ling H, Smith JD, Schott JM, Watkins LD, Lees AJ. Normal pressure hydrocephalus or progressive supranuclear palsy? A clinicopathological case series. J Neurol 2013;260(4):1009-1013

12 Tarnaris A, Toma AK, Pullen E, et al. Cognitive, biochemical, and imaging profile of patients suffering from idiopathic normal pressure hydrocephalus. Alzheimers Dement 2011;7(5): 501-508

13 Tarnaris A, Toma AK, Chapman MD, Keir G, Kitchen ND, Watkins LD. Use of cerebrospinal fluid amyloid-ßand total tau protein to predict favorable surgical outcomes in patients with idiopathic normal pressure hydrocephalus. J Neurosurg 2011;115(1):145-150

14 Tullberg M, Blennow K, Månsson JE, Fredman P, Tisell M, Wikkelsö C. Ventricular cerebrospinal fluid neurofilament protein levels decrease in parallel with white matter pathology after shunt surgery in normal pressure hydrocephalus. Eur J Neurol 2007;14(3):248-254 